\title{
On the nature of WO stars: a quantitative analysis of the WO3 star DR1 in IC $1613^{\star}$
}

\author{
F. Tramper ${ }^{1}$, G. Gräfener ${ }^{2}$, O. E. Hartoog ${ }^{1}$, H. Sana ${ }^{1}$, A. de Koter ${ }^{1,3}$, J. S. Vink ${ }^{2}$, L. E. Ellerbroek ${ }^{1}$, N. Langer ${ }^{4}$,

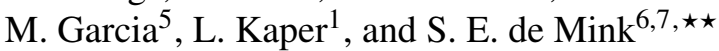 \\ 1 Astronomical Institute "Anton Pannekoek”, University of Amsterdam, Science Park 904, PO Box 94249, 1090 GE Amsterdam, \\ The Netherlands \\ e-mail: F.Tramper@uva.nl \\ 2 Armagh Observatory, College Hill, BT61 9DG Armagh, UK \\ 3 Instituut voor Sterrenkunde, Universiteit Leuven, Celestijnenlaan 200 D, 3001 Leuven, Belgium \\ 4 Argelander Institut für Astronomie, University of Bonn, Auf dem Hügel 71, 53121 Bonn, Germany \\ 5 Centro de Astrobiologia, CSIC-INTA, Ctra. Torrejon a Ajalvir km.4, 28850 Madrid, Spain \\ ${ }^{6}$ Observatories of the Carnegie Institution for Science, 813 Santa Barbara St, Pasadena, CA 91101, USA \\ 7 Cahill Center for Astrophysics, California Institute for Technology, Pasadena, CA 91125, USA
}

Received 27 June 2013 / Accepted 8 October 2013

\begin{abstract}
Context. Oxygen sequence Wolf-Rayet (WO) stars are thought to represent the final evolutionary stage of the most massive stars. The characteristic strong O vi emission possibly originates from an enhanced oxygen abundance in the stellar wind. Alternatively, the $\mathrm{O}$ vi emission can be caused by the high temperature of these stars, in which case the WO stars are the high-temperature extension of the more common carbon sequence Wolf-Rayet (WC) stars.

Aims. By constraining the physical properties and evolutionary status of DR1, a WO star in the low-metallicity Local Group dwarf galaxy IC 1613 and one of only two objects of its class known in a SMC-like metallicity environment, we aim to investigate the nature of WO stars and their evolutionary connection with WC stars.

Methods. We use the non-local thermodynamic equilibrium atmosphere code CMFGEN to model the observed spectrum of DR1 and to derive its stellar and wind parameters. We compare our values with other studies of WC and WO stars, as well as with the predictions of evolutionary models. We also model the surrounding nebula using the photo-ionization code cLoudy.

Results. The oxygen and carbon abundances that we obtain are comparable to values found for WC stars. The temperature and luminosity are, however, higher than those of WC stars. DR1 is embedded in the hottest known H in region in the Local Group. The nebular properties can be consistently reproduced by cLoudy models adopting DR1 as central ionizing source.

Conclusions. Comparison of the abundances and temperature of DR1 with core helium-burning models show that DR1 is currently well into the second half of helium burning. If the properties of DR1 are representative for the WO class, it would imply that WO stars are the high-temperature and high-luminosity extension of the WC stars, and do not necessarily represent a later evolutionary stage.
\end{abstract}

Key words. stars: Wolf-Rayet - stars: massive - stars: individual: DR1 - galaxies: individual: IC 1613 - HII regions

\section{Introduction}

The oxygen sequence Wolf-Rayet (WO) stars, introduced by Barlow \& Hummer (1982), are extremely rare. Only eight members of this class are currently known: Sand 4 (WR 102), Sand 5 (WR 142), MS4 (WR 30a) and WR 93b in the Milky Way, Sand 2 (BAT 99-123) and the recently discovered LH41-1042 (Neugent et al. 2012) in the Large Magellanic Cloud (LMC), Sand 1 (Sk 188) in the Small Magellanic Cloud (SMC), and DR1 in IC 1613. Their spectra are characterized by strong emission lines of highly ionized oxygen, in particular the O vi 3811-34 line with an equivalent width of up to $1700 \AA$ (Kingsburgh et al. 1995).

The origin of the high-excitation oxygen emission is widely attributed to the surfacing of this species during the late stages

\footnotetext{
* Based on observations obtained at the European Southern Observatory under GTO program 088.D-0181.

$\star \star$ Einstein Fellow.
}

of core helium (or possibly carbon) burning (Barlow \& Hummer 1982; Smith \& Maeder 1991). Revealing the core at this late stage of evolution requires the stellar mass-loss rate in prior stages to be relatively low. In this scenario the presence of WO stars is therefore preferred in low-metallicity environments (Smith \& Maeder 1991; Georgy et al. 2009), where the radiationdriven winds of their progenitors are relatively weak due to the low metal content (Vink et al. 2001; Vink \& de Koter 2005).

Crowther et al. (1998) introduced a quantitative classification scheme for the carbon sequence Wolf-Rayet (WC) and WO stars, in which the WO stars are the high-temperature extension of the WC class. In this classification, the highly ionized oxygen emission is primarily the result of excitation effects, and a significant abundance difference with the WC stars is not necessarily implied.

WR stars may be subject to sub-photospheric inflation of their stellar envelopes, resulting in lower stellar temperatures 
(Gräfener et al. 2012). As this effect is expected to be more pronounced at high metallicity (Ishii et al. 1999), WR stars in low-metallicity environments are expected to have higher stellar temperatures than those in the Galaxy.

WO stars are often thought to represent the final stage in the evolution of stars initially more massive than $25 M_{\odot}$ (Meynet \& Maeder 2003), i.e. including very massive stars that may avoid a red supergiant phase. If this is the case, WO stars offer the rare opportunity to study such stars just prior to their supernova (SN) explosions. Moreover, these SNe may be quite exotic, including helium-poor type Ic SNe, hypernovae (e.g., Nomoto et al. 2010), and, if they retain a rapidly rotating core, even gamma-ray bursts (Georgy et al. 2009; Woosley \& Bloom 2006; Yoon et al. 2012).

The surface abundances of early WC and WO stars closely reflect the core abundances. Measuring these abundance can thus provide constraints on the controversial ${ }^{12} \mathrm{C}(\alpha, \gamma){ }^{16} \mathrm{O}$ thermonuclear reaction rate.

In this work, we present a quantitative spectroscopic analysis of DR1 that allows us to constrain its physical and wind parameters, to investigate the nature of the object, and ultimately, its evolutionary stage. Located in the low-metallicity Local Group dwarf galaxy IC 1613, DR1 is one of the two WO stars known in a SMC-like metallicity environment (with $Z_{\mathrm{SMC}}=1 / 5 Z_{\odot}$ ). Metallicity estimates for IC 1613 range from $1 / 10 Z_{\odot}$ based on oxygen (e.g. Bresolin et al. 2007) to $1 / 5 Z_{\odot}$ based on iron (Tautvaišienè et al. 2007). DR1 thus offers a unique probe of the final evolutionary stages of massive stars at low metallicity.

The layout of this paper is as follows. Section 2 summarizes previous research on DR1. Section 3 describes the observations and data reduction. In Sect. 4 we analyze the stellar and nebular spectra, and in Sect. 5 we discuss DR1's properties, initial mass, evolutionary history and its eventual fate. Finally, Sect. 6 conveys our conclusions.

\section{Literature overview}

DR1 was discovered in 1982 by D’Odorico \& Rosa (1982). Based on spectra in the 4000-7000 $\AA$ range, they classified it as a peculiar WC star or a WC $+\mathrm{WN}$ binary. In the same year, spectra extending below $3600 \AA$ were obtained by Davidson \& Kinman (1982), who suggested that the star could be a member of the WO class because of the presence of strong $\mathrm{O}$ vi $\lambda \lambda 3811$ 34 emission. Davidson \& Kinman (1982) derived a temperature of $100 \mathrm{kK}$ for the star based on the nebular He II $\lambda 4686$ flux and assuming a blackbody distribution for the ionizing radiation. Subsequent studies by Armandroff \& Massey (1991) and Garnett et al. (1991) yielded spectral types of WC4-5 and WO4, respectively. The latter authors estimated that the effective temperature $T_{\text {eff }}$ should be in the range of $75 \mathrm{kK}$ to $90 \mathrm{kK}$ to reproduce the ionizing flux implied by the nebular $\mathrm{H} \beta$ and $\mathrm{He}$ II $\lambda 4686$ lines. Finally, DR1 and its surrounding nebula were intensively studied by Kingsburgh \& Barlow (1995), who adopted the spectral type WO3 (Kingsburgh et al. 1995). They derived $T_{\text {eff }}=$ $75 \mathrm{kK}$, a stellar luminosity $L=10^{6} L_{\odot}$, and a terminal wind velocity $v_{\infty}=2850 \mathrm{~km} \mathrm{~s}^{-1}$. They reported number abundances of $\mathrm{X}(\mathrm{C})=0.47, \mathrm{X}(\mathrm{O})=0.27$ and $\mathrm{X}(\mathrm{He})=0.25$, in agreement with the values that they found for other WO stars (Kingsburgh et al. 1995).

DR1 is the ionizing source of the surrounding $\mathrm{H}$ II region S3 (e.g., D'Odorico \& Rosa 1982), which exhibits unusually strong He II emission. Kingsburgh \& Barlow (1995) derived an electron temperature $T_{\mathrm{e}}=17.1 \mathrm{kK}$ and an oxygen abundance $12+\log (\mathrm{O} / \mathrm{H})=7.7$, or $Z=0.1 Z_{\odot}$ for $\mathrm{S} 3$ (with
Table 1. Journal of observations.

\begin{tabular}{lcc}
\hline \hline HJD & $t_{\exp }$ & $R$-band Seeing \\
At start of obs. & $(\mathrm{s})$ & $\left({ }^{\prime}\right)$ \\
\hline 2455857.705 & $2 \times 3600$ & $0.6-0.7$ \\
2455858.588 & 3600 & $0.8-0.9$ \\
2455859.642 & 1800 & 0.9 \\
\hline
\end{tabular}

$12+\log (\mathrm{O} / \mathrm{H})_{\odot}=8.69 ;$ Asplund et al. 2009). This makes the surrounding $\mathrm{H}$ II region one of the hottest known in the Local Group.

\section{Observations and data reduction}

\subsection{Spectroscopy}

We have observed DR1 using the X-Shooter spectrograph (Vernet et al. 2011) on ESO's Very Large Telescope (VLT), which covers a wavelength range extending from the near-UV to the near-IR (3000-25000 $\AA$ ). The observations were carried out in October 2011 during dark time, as part of the NOVA program for X-Shooter guaranteed time. A total of $3.5 \mathrm{~h}$ of integration on target has been obtained over three consecutive nights (see Table 1).

The selected slit widths of $0.8^{\prime \prime}, 0.9^{\prime \prime}$ and $0.9^{\prime \prime}$ result in a spectral resolving power of $6200,8800,5300$ in the UVB, VIS and NIR instrument arms, respectively. For the NIR, a $K$-band blocking filter has been used to avoid reflection of both sky background and object photons from this band into the 10000-20000 A region, optimizing the signal-to-noise ratio in the latter wavelength range.

To correct for instrument flexures, calibration frames have been taken before the start of each observation and after one hour of observations at the first night. Spectrophotometric standard stars have been observed at the beginning of each night to allow relative flux calibration.

The data have been reduced using the X-Shooter instrument pipeline version 1.3.7 (Modigliani et al. 2010). The extracted spectra were binned to $0.2 \AA$ in the UVB and VIS arms, and $0.6 \AA$ in the NIR arm. The (relative) flux-calibrated spectra for each observing block were co-added to obtain the final relative flux-calibrated spectrum.

\section{Quantitative spectroscopic analysis}

\subsection{Photometry}

The UBVRI magnitudes of the target (Table 2) were taken from the catalog of IC 1613's stellar population by Garcia et al. (2009). This catalog was built from PSF-photometry on multiple, dithered images of the irregular dwarf galaxy, taken with the Wide-Field Camera (WFC) at the $2.5 \mathrm{~m}$ Isaac Newton Telescope (INT). The set of broad-band filters used, Harris$B V R$ and $R G O-I U$, are similar to the Johnson's UBVRI system. The apparent and absolute magnitudes of DR 1 are presented in Table 2. The latter were computed adopting a foreground reddening of $E(B-V)=0.025$ (Schlegel et al. 1998) and a distance of $721 \mathrm{kpc}$ (Pietrzyński et al. 2006).

\subsection{Stellar spectrum}

We model the stellar spectrum of DR1 using the atmosphere code CMFGEN (Hillier \& Miller 1998). This code assumes a radial 
Table 2. INT/WFC UBVRI photometry.

\begin{tabular}{l|ccccc}
\hline \hline Quantity & $U$ & $B$ & $V$ & $R$ & $I$ \\
\hline$m$ & 18.543 & 19.877 & 19.857 & 19.827 & 19.901 \\
$\sigma_{m}$ & 0.006 & 0.006 & 0.009 & 0.010 & 0.015 \\
$M$ & -5.74 & -4.41 & -4.43 & -4.46 & -4.38 \\
\hline
\end{tabular}

Notes. Rows one and two provide the apparent magnitude $m$ and its error $\sigma_{m}$. The third row provides the corresponding absolute magnitude $M$, calculated adopting a distance of $721 \mathrm{kpc}$.

Table 3. Mass fractions of the elements in the model.

\begin{tabular}{lc}
\hline \hline Element & Mass fraction \\
\hline Neon & $1.74 \times 10^{-3}$ \\
Silicon & $6.99 \times 10^{-5}$ \\
Phosphorus & $6.12 \times 10^{-7}$ \\
Sulfar & $3.82 \times 10^{-5}$ \\
Chorine & $7.87 \times 10^{-7}$ \\
Argon & $1.02 \times 10^{-5}$ \\
Calcium & $6.44 \times 10^{-6}$ \\
Chromium & $1.70 \times 10^{-6}$ \\
Manganese & $9.44 \times 10^{-7}$ \\
Iron & $1.36 \times 10^{-4}$ \\
Nickel & $7.32 \times 10^{-6}$ \\
\hline
\end{tabular}

outflow of material from the atmosphere, of which the density and velocity structure is prescribed. The gas is assumed to be in a state of non-local thermodynamic equilibrium (non-LTE). The atomic models include both explicit levels and super-levels, and are of such complexity that effects such as back-warming and line-blanketing are self-consistently treated. Convergence to a solution that fulfills radiative equilibrium cannot be achieved by starting from a simple (e.g. gray LTE) solution and setting up a grid scanning the appropriate part of parameter space. Instead it requires one to migrate through a series of educated steps in specific parameters, from an existing model with fairly similar parameters to the final model.

This fitting procedure prevents a systematic search of parameter space and complicates an assessment of the uncertainties in the parameters of the final model. Furthermore, no specific diagnostic reacts exclusively to a given parameter, safe for luminosity which is determined from photometry. We therefore only specify error bars for this parameter. For the other parameters we give an indication of the accuracy of the obtained value. The steps taken to arrive at the final model are discussed below.

In our atmosphere models, the abundances of all modeled elements except hydrogen, helium, carbon, oxygen and neon are set to a value of $10 \%$ of solar (Table 3 ). The neon abundance is enhanced by the conversion of nitrogen into ${ }^{22} \mathrm{Ne}$ at the beginning of helium burning. The enhancement is predicted to be 1.4 times the initial oxygen mass fraction (Maeder 1983). For an oxygen abundance of $1 / 10$ solar, this results in a neon mass fraction very close to the solar value, which we therefore adopt. The hydrogen abundance is set to zero. The abundances of carbon and oxygen are being fitted and are not listed in Table 3. A summary of the ionization stages of each element included in the model is given in Table A.1.

Although we set the metallicity of our models to $1 / 10 Z_{\odot}$, which is the metallicity based on the oxygen abundance (e.g., Kingsburgh \& Barlow 1995; Bresolin et al. 2007), there are indications that IC 1613 may have a non-solar abundance pattern. Tautvaišienè et al. (2007) derived an iron abundance of $\log ([\mathrm{Fe} / \mathrm{H}]) \approx-0.7$ from the analysis of three M-type supergiants, i.e. very close to the SMC iron abundance. As iron is an important driver of WR winds, we also ran a model with $Z=1 / 5 Z_{\odot}$ to assess the impact of a higher metallicity on the derived parameters (Sect. 4.2.6).

We estimate the reddening of the DR1 source to be $E(B-$ $V)=0.05$ by adopting the extinction law of Fitzpatrick (1999) and dereddening the flux-calibrated spectrum to the slope of our model continuum. We normalize the dereddened spectrum by dividing by the model continuum, and setting the flux equal to unity at $6000 \AA$. The normalized spectrum does not show a noticeable slope, implying that the adopted value for the reddening is satisfactory. The best-fitting spectrum is presented in Fig. 1, and its parameters are listed in Table 4.

\subsubsection{Wind parameters: terminal velocity, $\beta$ and clumping}

In WR spectra, the full-width at half-maximum (FWHM) of the spectral lines represents the terminal velocity of the wind $\left(v_{\infty}\right)$, and this parameter can therefore easily be constrained. The bestfitting value is $v_{\infty}=2750 \mathrm{~km} \mathrm{~s}^{-1}$, and is accurate to within $50 \mathrm{~km} \mathrm{~s}^{-1}$.

The wind acceleration is assumed to be described by a single- $\beta$ velocity law, i.e.

$v(r)=v_{\infty}\left(1-\frac{R_{*}}{r}\right)^{\beta}$.

The value of $\beta$ influences the strength of the mass-loss sensitive lines (mainly O IV $\lambda 3300$ and C IV 25800 ), and is therefore degenerate with the wind strength for these lines. However, the value of $\beta$ also influences the shape of the optically thin He II lines formed at a larger radius. For low values of $\beta$ (i.e., high wind acceleration), the velocity gradient in the outer parts of the wind where these lines are formed is small, as the wind has already approached $v_{\infty}$. This causes flat-topped line profiles. For higher values of $\beta$, the wind is still accelerating toward $v_{\infty}$, and the velocity gradient at the line-forming region is larger, causing the line profiles to be more triangular-shaped (see Fig. 2). This diagnostic is somewhat degenerate with temperature (see Sect. 4.2.2), which affects the region of the wind in which these lines are formed. For higher temperatures the lines are formed further out in the wind where the velocity gradient again is smaller, thus producing flat-topped line profiles.

The shape of the He II and C IV line complex around $5400 \AA$ is affected by the adopted value of $\beta$. This line complex is also used to constrain the carbon abundance (see Sect. 4.2.3). The model has thus been iterated for different values of $\beta$, temperature and carbon abundance.

The shape of the line profiles of He II $\lambda 4859$ and He II $\lambda 6560$, as well as the shape of the He II and C IV complex at $5400 \AA$ have been used to determine the best-fit value for $\beta$. Figures 2 to 4 show the behavior of these lines for different sets of parameters. The parameters that are not explicitly specified in these plots are conform to the numbers given in Table 4. All diagnostics point toward a high value of $\beta=2$, i.e. a slowly accelerating wind, but lower values of $\beta$ are not excluded. A high value of $\beta(1.5-2)$ is consistent with theoretical predictions for optically thick stellar winds (Vink et al. 2011).

The value of the clumping parameter $f_{\mathrm{c}}$ influences the shape of the electron-scattering wings of the strong lines (see Fig. 5), 

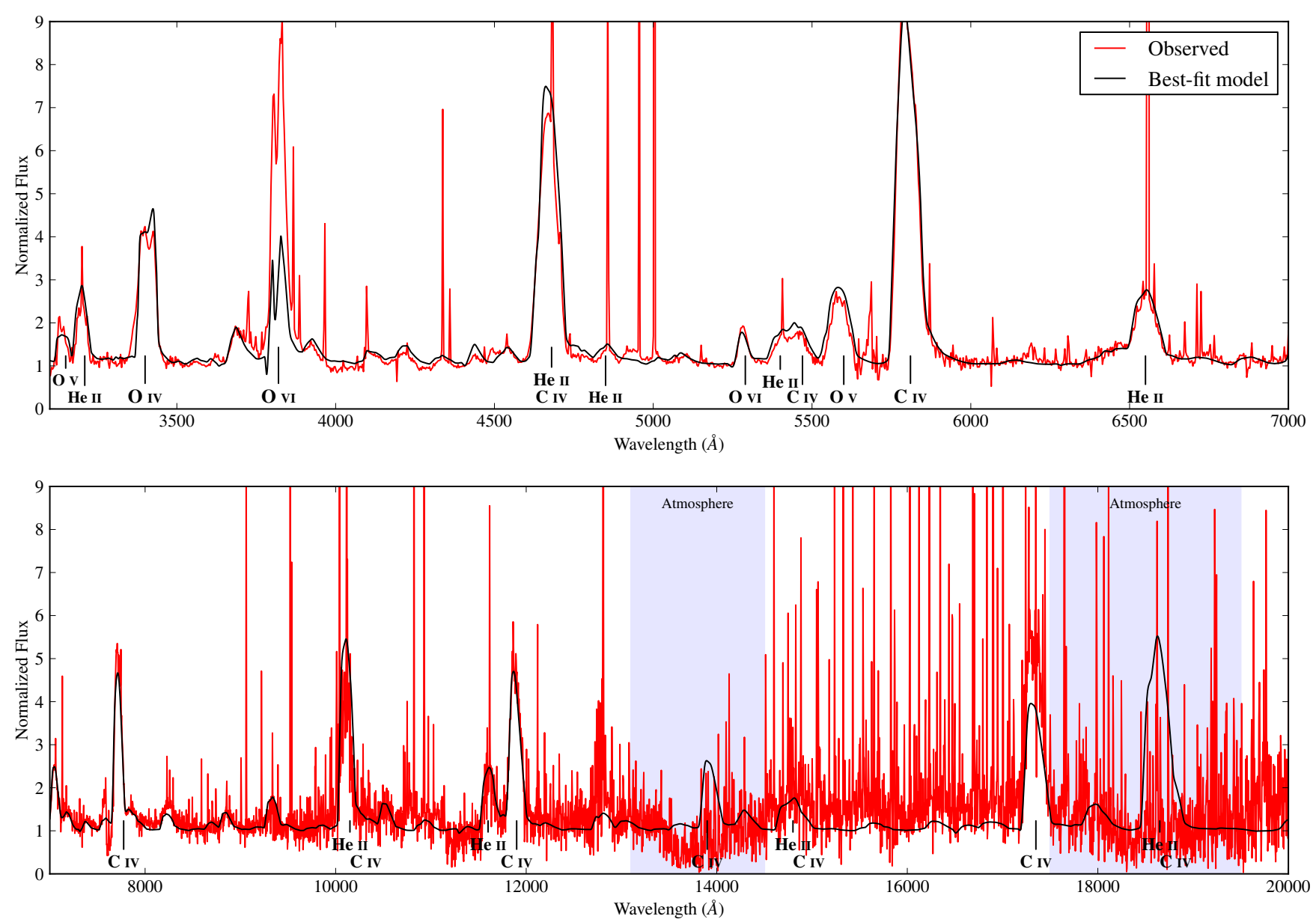

Fig. 1. Optical/near-infrared spectrum of DR1 (red). The best-fitting model spectrum is shown in black. As discussed in the text, the O vi $\lambda \lambda 3811$ 34 emission is underpredicted by the model. The shaded areas indicate the regions where the atmosphere of the Earth is opaque.

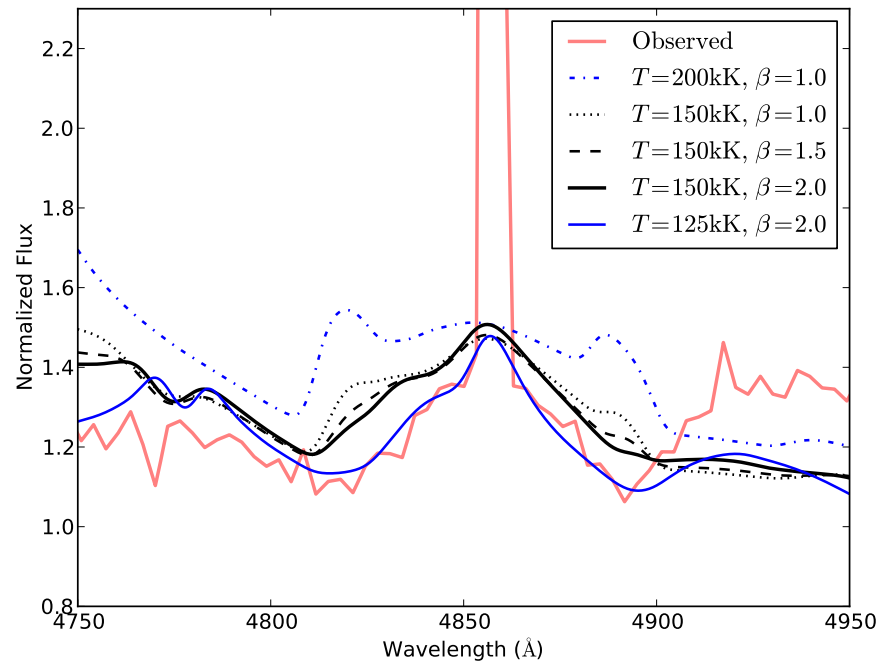

Fig. 2. Behavior of the He II line at $4859 \AA$ for different values of the temperature and $\beta$.

although the effect is weak. The commonly adopted value of $f_{\mathrm{c}}=$ 0.1 is in agreement with the observed spectrum, but cannot be well constrained. However, very weak clumping $\left(f_{\mathrm{c}} \geq 0.5\right)$ can be excluded, as O vi $\lambda 5290$ then gets reabsorbed in the wind and can no longer be fitted. Further constraints on the clumping are discussed in Sect. 5.2.

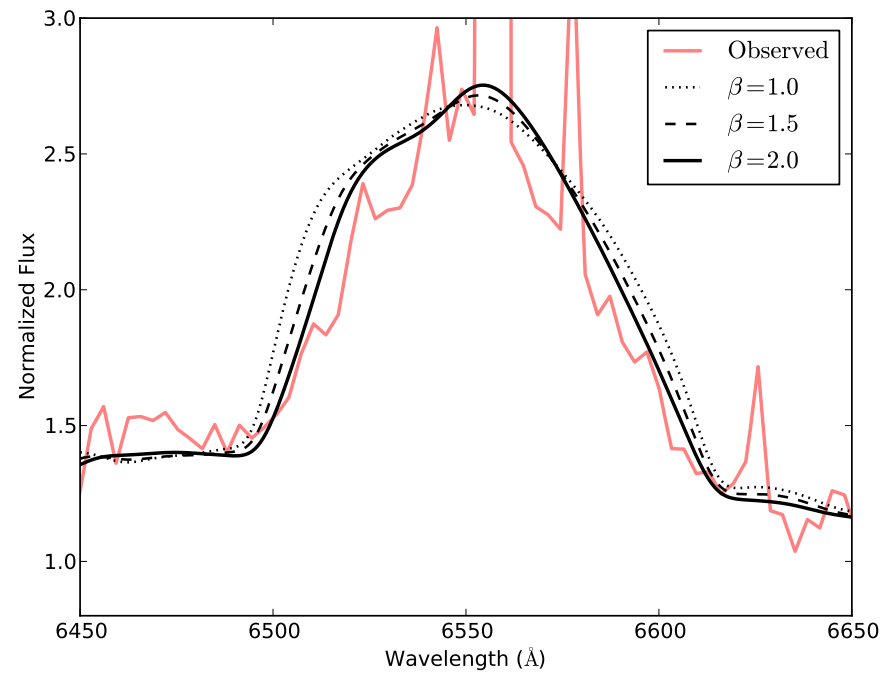

Fig. 3. Behavior of the Hе пा $\lambda 6560$ line for different values of $\beta$.

\subsubsection{Temperature}

Because the stellar wind is optically thick, we do not define the effective temperature at a Rosseland optical depth of $\tau_{\mathrm{R}}=$ $2 / 3$, as the corresponding radius is located far out in the wind. Instead, we define $T_{*}$ to represent the effective temperature at $\tau_{\mathrm{R}}=20$. This allows for a more meaningful comparison to 


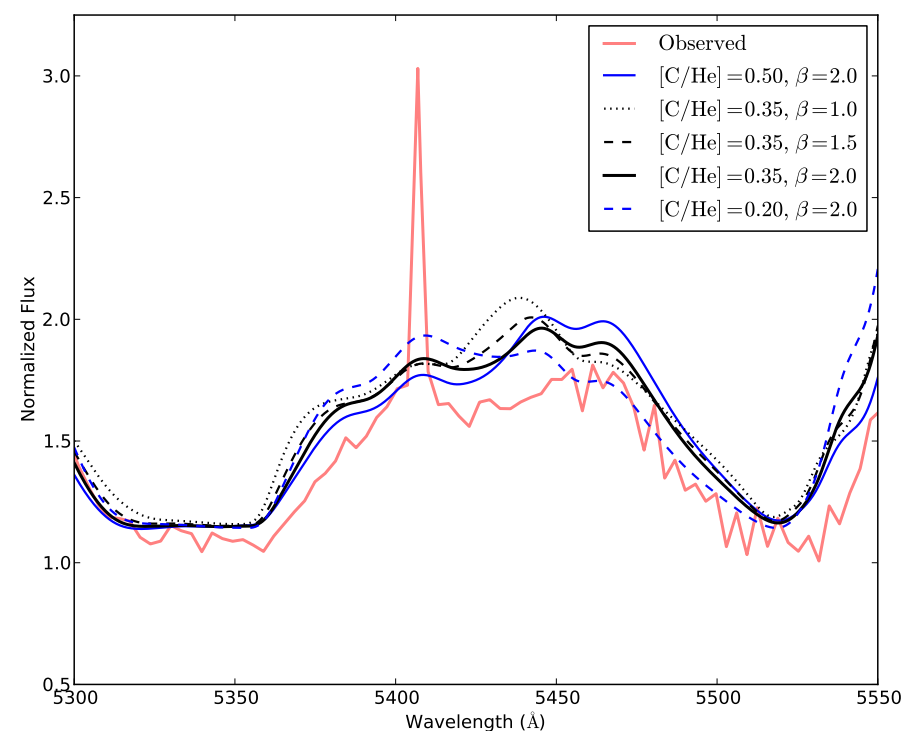

Fig. 4. Behavior of the He II and $\mathrm{C}_{\text {IV }}$ line complex around $5400 \AA$ for different values of $\beta$ and carbon abundance.

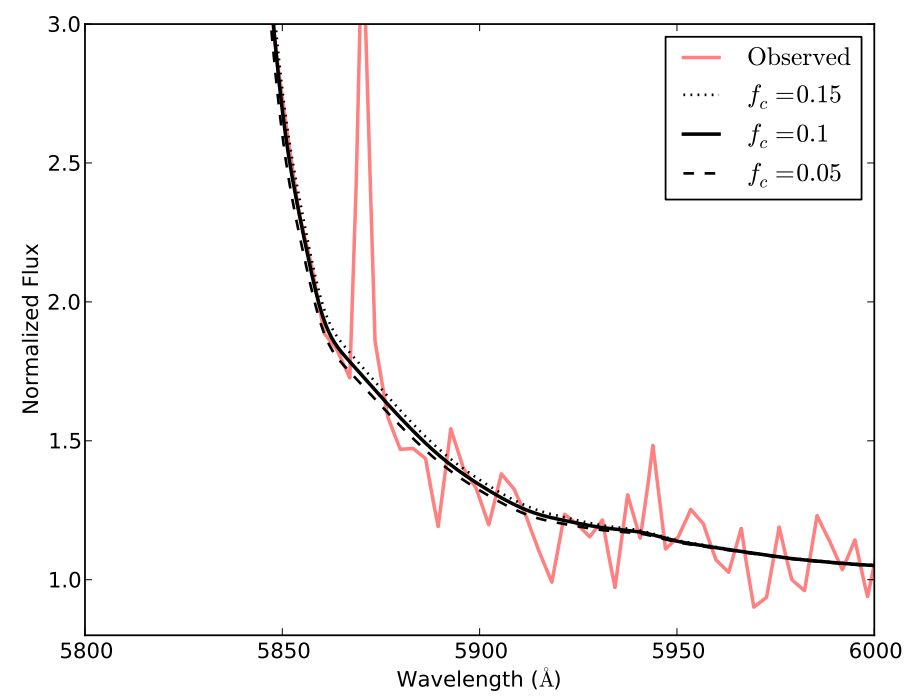

Fig. 5. Influence of the volume filling factor on the electron scattering wing of $C_{\text {Iv } \lambda \lambda 5801-12 .}$

evolutionary tracks, where the adopted effective temperature is not corrected for the presence of a wind.

Models have been calculated for temperatures $T_{*}$ ranging between $125 \mathrm{kK}$ and $200 \mathrm{kK}$. The line ratios of the different ionization stages of carbon and oxygen do not change significantly in most of the temperature range, and thus cannot be used to constrain the temperature. The presence of the strong $\mathrm{O}$ vi $\lambda \lambda 3811-$ 34 emission can be seen as an indication of a high temperature, although the full strength of the line cannot be reproduced. However, the line shapes of the optically thin He II lines are inconsistent with a very high temperature (see Fig. 2), as the model line profiles for these temperatures show a flat-topped shape, while the observed lines are triangular shaped (see Sect. 4.2.1).

A temperature of $150 \mathrm{kK}$ produces the best-fitting model to all lines except $\mathrm{O}$ vi $\lambda \lambda 3811-34$. The underpredicted flux in this line will be further discussed in Sect. 5.1. Although a lower temperature $(125 \mathrm{kK})$ provides an even better fit to the He II lines
Table 4. Best-fit parameters and ionizing fluxes.

\begin{tabular}{lc}
\hline \hline Parameter & Value \\
\hline$v_{\infty}$ & $2750 \mathrm{~km} \mathrm{~s}^{-1}$ \\
$\beta$ & 2.0 \\
$f_{\mathrm{c}}$ & 0.1 \\
$T_{*}$ & $150 \mathrm{kK}$ \\
{$[\mathrm{C} / \mathrm{He}]$} & 0.35 \\
{$[\mathrm{O} / \mathrm{He}]$} & 0.06 \\
$\dot{M} f_{\mathrm{c}}^{-0.5}$ & $5.6 \times 10^{-5} M_{\odot} \mathrm{yr}^{-1}$ \\
$\log \left(L / L_{\odot}\right)$ & $5.68 \pm 0.10$ \\
\hline $\log \left(Q_{0}\right)$ & $49.5 \mathrm{~s}^{-1}$ \\
$\log \left(Q_{1}\right)$ & $49.3 \mathrm{~s}^{-1}$ \\
$\log \left(Q_{2}\right)$ & $48.0 \mathrm{~s}^{-1}$ \\
\hline
\end{tabular}

Notes. $Q_{0}, Q_{1}$ and $Q_{2}$ are the number of ionizing photons per second for hydrogen, $\mathrm{He}_{\mathrm{I}}$ and $\mathrm{He}$ II, respectively. Except for the luminosity, the determination of formal error bars is not possible. Uncertainties on the parameters are discussed in the text.

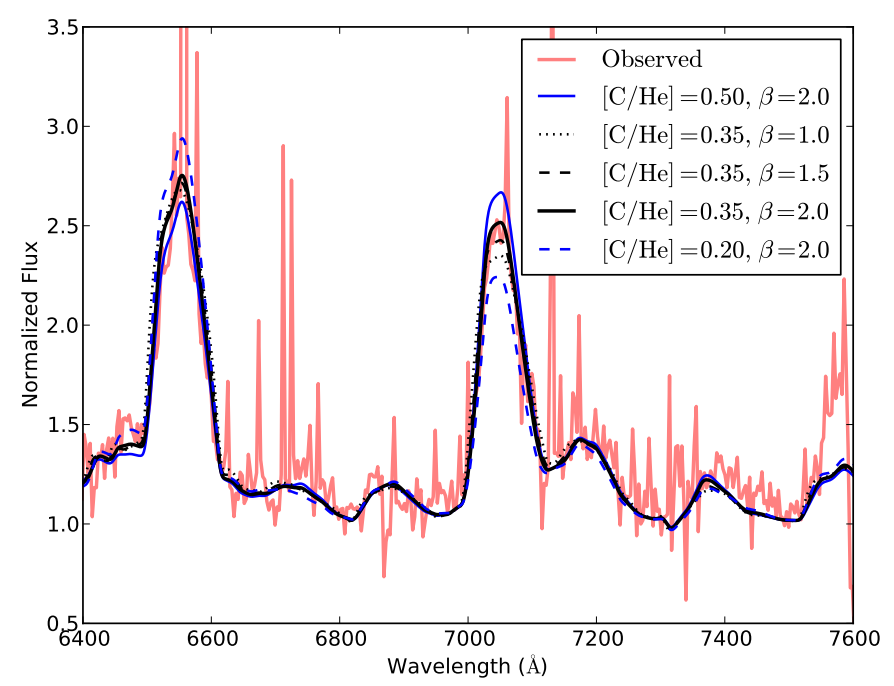

Fig. 6. Behavior of the $\mathrm{He}_{\text {II }} \lambda 6560$ and $\mathrm{C}_{\text {IV }} \lambda 7063$ lines for different values of $\beta$ and carbon abundance.

(see Fig. 2), the line ratio of $\mathrm{O} v \mathrm{vI} \lambda 5290$ to $\mathrm{O} v \lambda 5598$ changes significantly for this temperature, with too much emission in the O v line compared to O vI. We therefore adopt $150 \mathrm{kK}$ as bestfit value. Models with a temperature $T_{*}>175 \mathrm{kK}$ are excluded based on the He II line shapes (see Fig. 2).

\subsubsection{Carbon and oxygen abundances}

The carbon abundance (modeled as $[\mathrm{C} / \mathrm{He}]$ by number), has been determined using the He II $\lambda 5412$ and $\mathrm{C}$ IV $\lambda 5471$ line ratio. As can be seen from Fig. 4, this diagnostic is not very sensitive to the adopted value of $\beta=2$. For this $\beta$, changes in the $C_{\text {IV }}$ peak are minimal for different values of the carbon abundance; a value of $[\mathrm{C} / \mathrm{He}]=0.35$ agrees best with the overall spectrum (see Fig. 6 for the typical behavior of the carbon and helium lines for different carbon abundances).

The oxygen abundance ([O/He] by number) can be constrained with an accuracy of 0.01 by the strength of the $\mathrm{O}$ vi $\lambda 5290$ and $\mathrm{O} v \lambda 5598$ lines (see Fig. 7). We derive a value of $[\mathrm{O} / \mathrm{He}]=0.06$ as best-fitting abundance. 


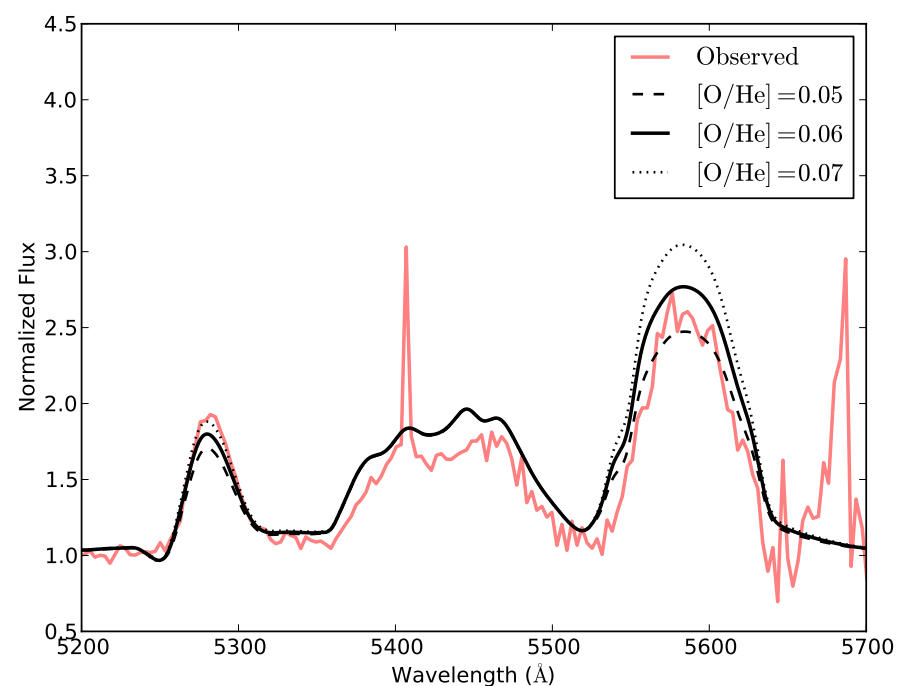

Fig. 7. Behavior of the O vi $\lambda 5290$ and Ov $\lambda 5598$ lines for different values of the oxygen abundance.

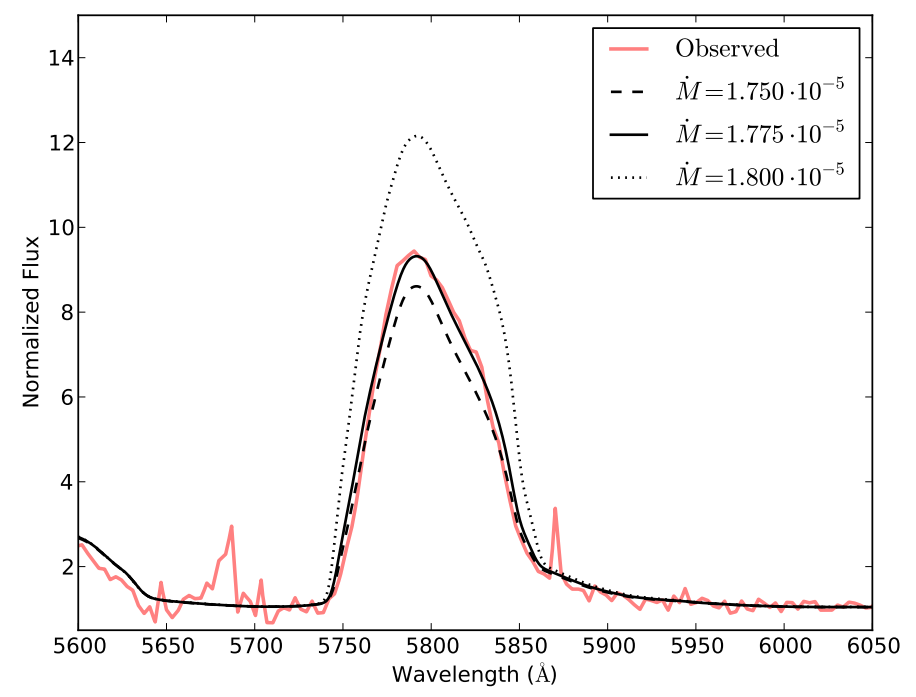

Fig. 8. Behavior of the $\mathrm{C}_{\text {Iv }}$ line at $\lambda \lambda 5801-12$ for different values of $\dot{M}$.

\subsubsection{Mass-loss rate}

The strength of the $\mathrm{C}_{\mathrm{IV}} \lambda \lambda 5801-12$ and $\mathrm{O}_{\mathrm{IV}} \lambda \lambda 3404-12$ lines are very sensitive to the mass-loss rate, and therefore these lines serve as the prime diagnostic for this parameter. In general, the equivalent width of WR emission lines is found to be invariant if the transformed radius

$R_{\mathrm{t}}=R_{*}\left[\frac{v_{\infty}}{2500 \mathrm{~km} \mathrm{~s}^{-1}} / \frac{\dot{M}}{\sqrt{f_{\mathrm{c}}} 10^{-4} M_{\odot} \mathrm{yr}^{-1}}\right]^{\frac{2}{3}}$

is kept fixed (Schmutz et al. 1989). The derived mass-loss rate is therefore dependent on the adopted $v_{\infty}, f_{\mathrm{c}}, L$ and $T_{*}$ (the last two values determining $R_{*}$ ).

For the parameters of our best-fitting model the mass-loss rate is $\dot{M}=1.8 \times 10^{-5} M_{\odot} \mathrm{yr}^{-1}$, and can be constrained to within 0.05 dex (see Fig. 8). The corresponding transformed radius is $R_{\mathrm{t}}=1.3 R_{\odot}$.
Table 5. Nebular line ratios relative to $\mathrm{H} \beta$.

\begin{tabular}{|c|c|c|}
\hline $\begin{array}{c}\text { Line ID } \\
\lambda(\AA)\end{array}$ & Observed & $\begin{array}{r}\text { Cloudy } \\
\text { model }\end{array}$ \\
\hline \multicolumn{3}{|l|}{$\mathrm{He}_{\text {II }}$} \\
\hline 4686 & $0.51 \pm 0.03$ & 0.53 \\
\hline \multicolumn{3}{|l|}{$\mathrm{He}_{\mathrm{I}}$} \\
\hline 4471 & $0.017 \pm 0.007$ & 0.022 \\
\hline 5876 & $0.058 \pm 0.014$ & 0.058 \\
\hline 6678 & $0.017 \pm 0.007$ & 0.016 \\
\hline \multicolumn{3}{|l|}{$\left[\mathrm{O}_{\text {II }}\right]$} \\
\hline 3726 & $0.12 \pm 0.03$ & 0.11 \\
\hline 3729 & $0.18 \pm 0.04$ & 0.16 \\
\hline \multicolumn{3}{|l|}{ [O III] } \\
\hline 4363 & $0.13 \pm 0.01$ & 0.10 \\
\hline 4959 & $1.45 \pm 0.07$ & 1.39 \\
\hline 5007 & $4.36 \pm 0.18$ & 4.20 \\
\hline \multicolumn{3}{|l|}{$[\mathrm{Ne}$ III] } \\
\hline 3869 & $0.63 \pm 0.06$ & 0.40 \\
\hline 3968 & $0.19 \pm 0.08$ & 0.12 \\
\hline \multicolumn{3}{|l|}{ [Ar IV] } \\
\hline 4711 & $0.061 \pm 0.011$ & 0.058 \\
\hline 4740 & $0.053 \pm 0.019$ & 0.045 \\
\hline \multicolumn{3}{|l|}{$\left[\mathrm{S}_{\mathrm{II}}\right]$} \\
\hline 6716 & $0.052 \pm 0.020$ & 0.028 \\
\hline 6731 & $0.038 \pm 0.016$ & 0.020 \\
\hline \multicolumn{3}{|l|}{ [S III] } \\
\hline 6312 & $0.024 \pm 0.017$ & 0.008 \\
\hline
\end{tabular}

\subsubsection{Luminosity}

To determine the luminosity of DR1, we have computed synthetic UVRI magnitudes of a fitted model spectrum (with $L=3 \times 10^{5} L_{\odot}$ ) by integrating the model flux using the transmission functions of the filters as provided by the INT website ${ }^{1}$. Zero-point magnitudes were determined by performing spectrophotometry on a Kurucz model spectrum (Castelli \& Kurucz 2004) of Vega $\left(T_{\mathrm{eff}}=9500 \mathrm{~K}, \log g=4.0, d=7.68 \mathrm{pc}\right.$, $\left.R=2.5 R_{\odot}\right)$. A reddening of $E(B-V)=0.05$ (Sect. 4.2) is then added to the obtained values. Because the $\mathrm{O}$ vi $\lambda \lambda 3811-34$ emission is underpredicted by the model, we do not use the $B$-band magnitude, which is affected by the flux in this line. Scaling the synthetic magnitudes to match the observed values (Table 2) yields a luminosity of $\log \left(L / L_{\odot}\right)=5.68 \pm 0.10$. The error in this value is based on the spread in magnitude differences for each filter. The error induced by the uncertainty in the distance to IC 1613 (3\%; Pietrzyński et al. 2006) is in comparison negligable. Note that the luminosity is overpredicted if undetected companion stars are contributing to the observed flux. After determining the luminosity, the mass-loss rate is adjusted to fit the observed line strengths.

\subsubsection{Metallicity}

To determine the impact of the uncertainty in the metallicity of DR1, we computed a model with SMC metallicity $\left(Z=1 / 5 Z_{\odot}\right)$. Apart from the mass-loss rate, the derived parameters are not noticeably affected by this change. The mass-loss rate needed to fit the spectrum is slightly lower than the value derived for the lowmetallicity model $\left(\dot{M} f_{\mathrm{c}}^{-1 / 2}=5.1 \times 10^{-5} M_{\odot} \mathrm{yr}^{-1}\right.$, corresponding

\footnotetext{
1 http://catserver.ing.iac.es/filter/list.php? instrument $=\mathrm{WFC}$
} 
to $\dot{M}=1.6 \times 10^{-5} M_{\odot} \mathrm{yr}^{-1}$ for $\left.f_{\mathrm{c}}=0.1\right)$. This is likely the result of more efficient line blanketing in the high-metallicity model.

\subsection{Nebular spectrum}

DR1 is surrounded by the ionized nebula S3. The narrow nebular emission lines are clearly distinguishable from the Wolf-Rayet spectrum (e.g., Fig. 1). Apart from the lines usually seen in $\mathrm{H}_{\text {II }}$ regions, S3 exhibits strong He II emission (He II $\lambda 4686 / \mathrm{H} \beta=$ $0.51 \pm 0.03$ ), indicative of a hot ionizing source. The line strengths from a selection of emission lines relative to $\mathrm{H} \beta$ are given in Table 5. The errors on the values have been calculated by the method described in Hartoog et al. (2012). Based on the $\mathrm{X}$-Shooter acquisition image, we estimate the projected dimensions of the asymmetric nebula to be approximately $30 \times 60 \mathrm{pc}$.

Following Pagel et al. (1992), we derive an electron temperature $T_{\mathrm{e}}=17.5 \pm 0.6 \mathrm{kK}$ based on the nebular [O III] emission. This temperature is higher than measured in other $\mathrm{H}_{\text {II }}$ regions with $\mathrm{He}_{\text {II }}$ emission, making S3 the hottest $\mathrm{H}$ II region currently known in the Local Group (see Kehrig et al. 2011, for an overview of known He II nebulae). This electron temperature is indicative for both the high temperature of the ionizing source, and the low-metallicity environment.

The oxygen abundance derived from the [O II] and [O III] emission lines is $12+\log (\mathrm{O} / \mathrm{H})=7.56 \pm 0.11$. The electron density in the nebula is in the low density regime $\left(<75 \mathrm{~cm}^{-3}\right)$ based on the [O II] $\lambda 3729 / 3726$ and [S II] $\lambda 6716 / 6731$ line ratios (Osterbrock \& Ferland 2006).

Assuming that DR1 is the dominant ionizing source of S3, we aim to reproduce the observed nebular properties using the photo-ionization code Cloudy v8.0 (Ferland et al. 2013). To do this, we model the $\mathrm{H}_{\text {II }}$ region as a spherically symmetric cloud surrounding an ionizing source, for which we use the best-fit model of DR1. We derive nebular line strengths by computing the nebular spectrum in the line of sight toward the central source. In our models, the metal abundances in the cloud are set to $18 \%$ of the abundance pattern in the Orion nebula ${ }^{2}$, corresponding to $Z=0.15 Z_{\odot}$ based on oxygen. The Galactic foreground extinction is discussed in Sect. 4.1. There is no indication that the line of sight toward DR1 suffers from extinction in IC 1613. We therefore do not include dust grains in our model. The inner radius of the cloud $r_{0}$ is fixed to $0.1 \mathrm{pc}$, as varying this inner radius for reasonable values $\left(r_{0} \leq 1 \mathrm{pc}\right)$ does not change the resulting parameters significantly.

We assume the following density profile, containing both a $r^{-2}$ dependency and a constant component $n_{\mathrm{c}}$ :

$n(r)=n_{0}\left(1+\frac{r-r_{0}}{r_{\text {scale }}}\right)^{-2}+n_{\mathrm{c}}$,

where $n(0)+n_{\mathrm{c}}$ is the density at $r_{0}$ and $r_{\text {scale }}$ is the scale-length. We compute a grid of models varying $n_{0}, r_{\text {scale }}$, and $n_{\mathrm{c}}$. The line ratios of our best model are given in Table 5, and agree well with the observed values.

The adopted model has $n_{0}=35 \mathrm{~cm}^{-3}, r_{\text {scale }}=16 \mathrm{pc}$, and $n_{\mathrm{c}}=8 \mathrm{~cm}^{-3}$, although small variations in these parameters also give nebular properties that match the observed values. However, models with a constant density profile (i.e. $n_{0}=0$ ), as well as models with $r_{\text {scale }}<5 \mathrm{pc}$, cannot reproduce the observed properties.

\footnotetext{
2 The Orion nebula abundances used in Cloudy are a subjective mean of values derived by Baldwin et al. (1991), Rubin et al. (1991), Osterbrock et al. (1992) and Rubin et al. (1993).
}

These line ratios in Table 5 correspond to an electron temperature $T_{\mathrm{e}}=16.1 \mathrm{kK}$ and an oxygen abundance of $12+\log (\mathrm{O} / \mathrm{H})=$ 7.61. This abundance is consistent with the observed value, and would correspond to a metallicity of $Z \approx 0.08 Z_{\odot}$ based on [O II] and $[\mathrm{O}$ III $]$. As the oxygen abundance in our model is set to a value of $15 \%$ solar, this indicates that approximately half of the oxygen is in an ionization state higher than $\mathrm{O}$ III. This is confirmed by the inspection of the relative population of the oxygen ionization stages in our model. Finally, the Strömgren radius of the modeled $\mathrm{H}_{\text {II }}$ region is $15 \mathrm{pc}$, in agreement with the size of the observed nebula. The electron temperature is lower than observed, which is the case for all models that reproduce the line ratios well. This is likely caused by the assumed abundance pattern of the metals, which is observed to differ between individual H II regions (e.g., Zaritsky et al. 1994).

We also investigate the sensitivity of the nebular spectrum to changes in the stellar temperature, the parameter that mostly controls the production of ionizing photons. This sensitivity turns out to be very modest. Nebular models where we varied the temperature of the ionizing source by $\Delta T_{*}=25 \mathrm{kK}$ can also reproduce the observed nebular properties. The results of both the nebular and stellar analysis provide a consistent picture, suggesting that DR1 is indeed the main ionizing source of S3.

\section{Discussion}

\subsection{Oxygen abundance and temperature}

The prominent $\mathrm{O}$ vi $\lambda \lambda 3811-34$ emission is the tell-tale signature of WO stars. However, this line is notoriously difficult to reproduce by models of their atmospheres. Two modeling approaches can be followed. Either the focus is to reproduce the prominent $\mathrm{O}$ vi $\lambda \lambda 3811-34$ emission while accepting a poorer fit to the overall spectrum, or the aim is to reproduce the entirety of the spectrum, accepting a relatively poor fit to $\mathrm{O}$ vi $\lambda \lambda 3811-34$.

The modeling of the galactic WO stars WR102 and WR142 (both with spectral type WO2) by Sander et al. (2012) focusses on reproducing the $\mathrm{O}$ VI $\lambda \lambda 3811-34$ emission. This can be achieved by adopting a high temperature and an oxygen abundance twice as high as they on average find for early-type WC stars. However, the model spectrum underestimates the flux in some of the other lines seen in the observed spectrum, such as the $\mathrm{O} v \lambda 5598$ emission. This is likely due to the high adopted temperature, which causes the higher ionization states to be preferred.

Crowther et al. (2000) modeled the far-UV and optical spectrum of the LMC WO star Sand 2 taking the alternative approach, and in their model do not reproduce the $\mathrm{O}_{\mathrm{VI}} \lambda \lambda 3811$ 34 emission. Their obtained temperature is $50 \mathrm{kK}$ lower than temperatures obtained by Sander et al. (2012) who primarely modeled $\mathrm{O}$ vi $\lambda \lambda 3811-34$, although the difference can also be attributed to the difference in spectral type.

As our data cover a large wavelength range (3000$20000 \AA$ ), we focus on obtaining a good overall fit while neglecting the $\mathrm{O}_{\mathrm{VI}} \lambda \lambda 3811-34$ line. Although the strong $\mathrm{O}_{\mathrm{VI}}$ $\lambda \lambda 3811-34$ emission is underpredicted by a factor $2-3$, the strength of the O vi $\lambda 5290$ line is well represented by our models. This suggests the presence of an additional mechanism not accounted for in the modeling, which significantly contributes to the O vi $\lambda \lambda 3811-34$ emission.

The $\mathrm{O}_{\mathrm{VI}} \lambda \lambda 3811-34$ emission is particularly sensitive to temperature, oxygen abundance and wind strength. A higher temperature will increase the $\mathrm{O}$ vi to $\mathrm{O} v$ ionization ratio, producing stronger emission in $\mathrm{O}$ vI. A higher oxygen abundance 
A\&A 559, A72 (2013)

Table 6. Comparison of carbon and oxygen abundances measured in WC and WO stars.

\begin{tabular}{lllcccc}
\hline \hline Reference & Galaxy & Spectral type & \multicolumn{2}{c}{ Mass fraction } & \multicolumn{2}{c}{ Number abundance } \\
& & & C & O & C/He & O/He \\
\hline This work & IC 1613 & WO3 & 0.46 & 0.10 & 0.35 & 0.06 \\
Gräfener et al. (1998) & LMC & six WC4 & 0.4 & $0.1-0.3$ & & \\
Crowther et al. (2000) & LMC & WO & & & 0.7 & 0.15 \\
Crowther et al. (2002) & LMC & six WC4 & $0.2-0.4$ & 0.1 & $0.1-0.35$ & $\leq 0.06$ \\
Hillier \& Miller (1999) & MW & WC5 & & & 0.4 & 0.1 \\
De Marco et al. (2000) & MW & WC8 & & & 0.15 & 0.03 \\
Dessart et al. (2000) & MW & four WC & & & $0.08-0.25$ & $0.02-0.1$ \\
Gräfener et al. (2002) & MW & WC5 & 0.45 & 0.04 & & \\
Crowther et al. (2006) & MW & WC9 & & & 0.2 & 0.01 \\
Smartt et al. (2001) & M31 & WC6 & & & 0.1 & \\
\hline
\end{tabular}

will increase the strength of oxygen lines relative to that of lines of other elements. The dependence of the strength of $\mathrm{O}$ vi $\lambda \lambda 3811-34$ on the mass-loss rate is more subtle: as the O vi $\lambda \lambda 3811-34$ emission is formed in deep layers of the stellar wind, part of the emission is absorbed by the outer layers of the wind itself. A higher mass-loss rate, i.e. a denser wind, will therefore result in weaker observed emission of this line.

A physical motivation for the poor modeling of the $\mathrm{O}$ vi $\lambda \lambda 3811-34$ line may be found in its susceptibility to soft $\mathrm{X}$ ray emission at the base of the outflow, e.g. due to shocks in the wind-acceleration zone. Such shocks could pump the upper level through the $2 \mathrm{p}^{2} \mathrm{p}^{0}-3 \mathrm{~s}^{2} \mathrm{~s}$ transition at $184 \AA$. If the X-ray production is quite localized at the base of the wind, the layers above this zone may shield (part of) this X-ray emission. This prevents the C IV $\lambda \lambda 5801-12$ line, which originates from the same electron configuration transition as $\mathrm{O}$ VI $\lambda \lambda 3811-34$ but is formed further out in the wind, to react in a similar way.

Neglecting the O vi $\lambda \lambda 3811-34$ line, the carbon and oxygen abundances that we obtain are comparable to values found for early-type WC stars in a variety of environments (see Table 6). We thus conclude that the carbon and oxygen abundance in DR1 present no indication of enhancement compared to WC stars.

\subsection{Evolutionary state}

As most massive stars are formed in close binary systems (Sana et al. 2012, 2013), it is possible that DR1 has or has had a close companion star. If DR1 is part of a close binary, it is likely that mass transfer between the stars occurs at some point during the evolution of the system, influencing their evolution. For instance, if DR1 has transferred mass onto a companion star, less mass loss through a stellar wind is needed to expose the helium-burning products. Vice versa, if DR 1 has gained mass or is the product of a merger, this will likely have led to high rotation rates (e.g., de Mink et al. 2013). This may lead to enhanced mass-loss due to rotation and rotational mixing, also making it easier for the helium-burning products to surface. Because we have no indication for binarity of DR1, we limit the discussion of its evolutionary state to a single-star perspective. However, we cannot exclude the possibility that DR1 is the product of binary interaction.

Figures 9 and 10 show the position of DR1 in the Hertzsprung-Russell diagram (HRD). For comparison, the two Galactic WO stars and the WC stars analyzed by Sander et al. (2012), and the WO star analyzed by Crowther et al. (2000) are also plotted. Both DR1 and the two WO2 stars from Sander et al. (2012) are positioned at the high temperature and high

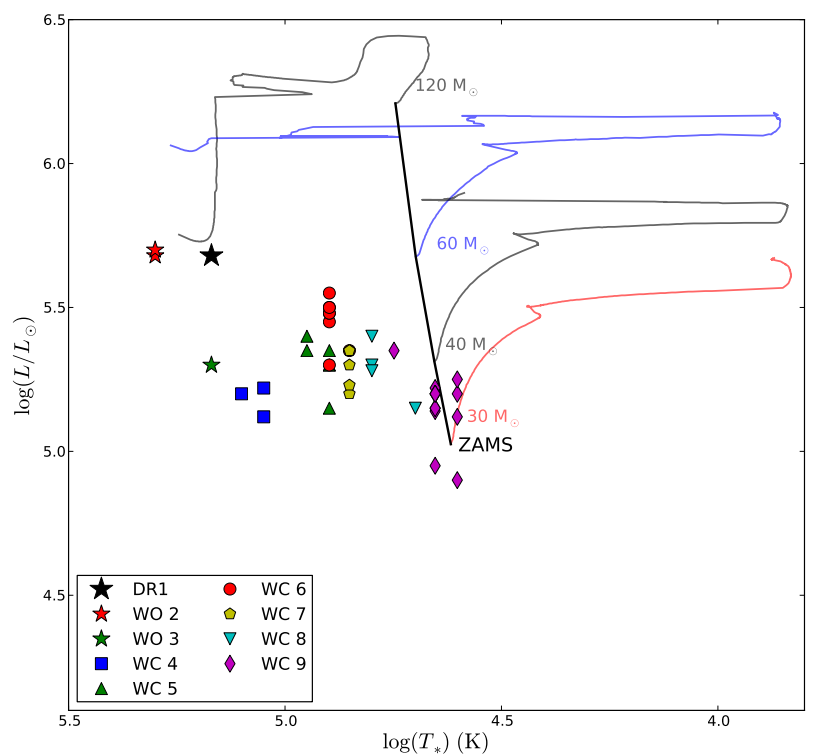

Fig. 9. Location of DR1 in the Hertzsprung-Russell Diagram. Also plotted are the WO2 and WC stars analyzed by Sander et al. (2012), the LMC WO3 star analyzed by Crowther et al. (2000) and evolutionary tracks for SMC metallicity from Meynet \& Maeder (2005) for an initial rotation rate of $300 \mathrm{~km} \mathrm{~s}^{-1}$.

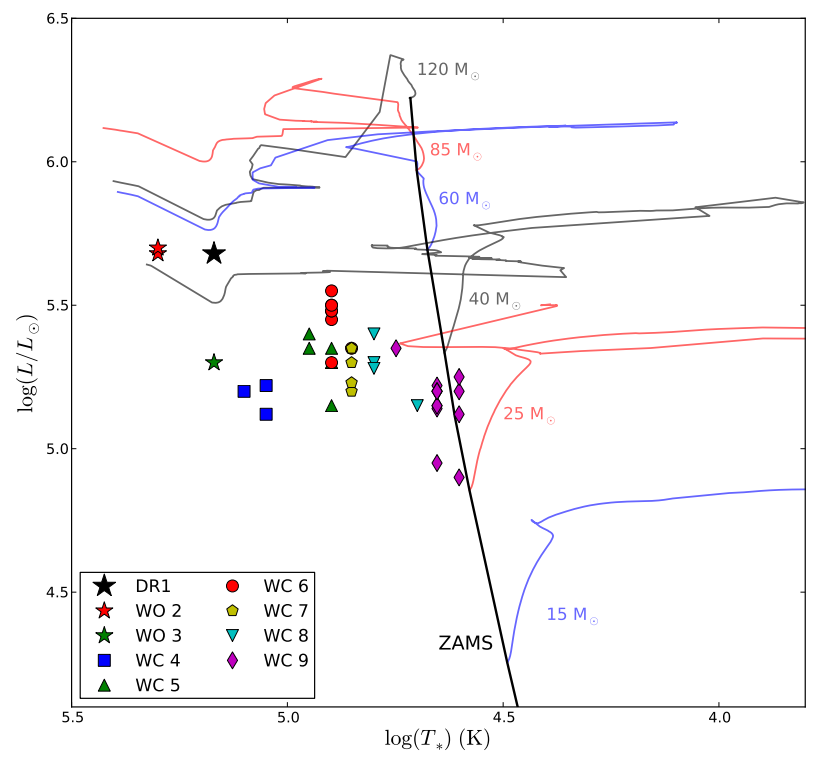

Fig. 10. Same as Fig. 9, but with evolutionary tracks for Galactic metallicity from Ekström et al. (2012) for an initial rotation rate of $0.4 v_{\text {crit }}$. 
luminosity regime of the strip in the HRD occupied by the WC and WO stars. The LMC WO3 star Sand 2 from Crowther et al. (2000, for which the luminosity is much less uncertain than the Galactic cases due to the better constrained distance) is considerably less luminous, while having the same temperature as DR1. Both DR1 and Sand 2 are located very close to the helium ZAMS, indicating that they could be the descendants of stars with a different initial mass. Alternatively, it is possible that the luminosity of DR1 is overestimated if unseen companions contribute significantly to the observed flux.

Figures 9 and 10 also show the evolutionary tracks from Meynet \& Maeder (2005) and Ekström et al. (2012), representing single stars with SMC metallicity initially rotating at $300 \mathrm{~km} \mathrm{~s}^{-1}$ and single stars with Galactic metallicity initially rotating at $40 \%$ of critical, respectively. Figure 9 shows that DR 1 is located at a position in the HRD that is coinciding with the track for the late stages of evolution of a $120 M_{\odot}$ star at SMC metallicity. The corresponding current-day mass would be $\approx 18 M_{\odot}$. This is consistent with the mass of $20 M_{\odot}$ that is computed from the mass-luminosity relation of Gräfener et al. (2011). A firm determination of the initial mass of DR 1 cannot be made, however, as the track for stars with initial masses higher than $60 M_{\odot}$ also reaches the high-temperature domain of the HRD. Uncertainties that may complicate an identification of the evolutionary stage of DR1 from these tracks include the initial rotational velocity, metal content and the mass-loss properties throughout the different evolutionary phases (in particular during the luminous blue variable and red supergiant phases). In general, it is thought that higher rotation rates lead to a higher mass-loss rate, and therefore to lower stellar masses during the WC or WO stage. In specific cases, however, this trend may be broken (see Meynet \& Maeder 2003).

For a Galactic environment, Fig. 10 shows that evolutionary tracks for initial masses higher than $40 M_{\odot}$ reach the hightemperature domain of the HRD where the WO stars are located. Although suffering from the same uncertainties as listed above, it suggests that the occurrence of WO stars is slightly favored at higher metallicities, as the mass range for potential progenitors is larger. This is in contrast with predictions based on the assumption that the oxygen abundance is enhanced in WO stars, in which case their formation is favored in sub-Galactic metallicity environments (see, e.g., Georgy et al. 2009, their Fig. 1).

Figure 11 compares the carbon and oxygen abundances of DR1 to evolutionary predictions of the change of the helium, carbon and oxygen abundances during core-helium burning. While low-metallicity models have been used for this comparison, the influence of the metallicity is negligible. Higher masses, however, lead to a markedly lower carbon abundance and correspondingly higher oxygen abundance at a given helium mass fraction. Note that only two of the three abundances are independent, as the sum of all three is very close to one.

The ${ }^{12} \mathrm{C}(\alpha, \gamma){ }^{16} \mathrm{O}$ thermonuclear reaction rate used in these models is still highly uncertain (Tur et al. 2007). For instance, a higher ${ }^{12} \mathrm{C}(\alpha, \gamma){ }^{16} \mathrm{O}$ cross section would lead to somewhat lower carbon abundances at a given helium mass fraction. The reaction rate employed in the models shown in Fig. 11 is the one proposed by Weaver \& Woosley (1993), which appears to be required for massive stars to reproduce the Solar abundance pattern between oxygen and calcium. If this rate is correct, Fig. 11 indicates that DR1 is likely not the descendant of a star of initially several $100 M_{\odot}$.

From Fig. 11, we also see that the surface composition of DR1 corresponds to material that was in the core of the star roughly half-way into core-helium burning. This implies that

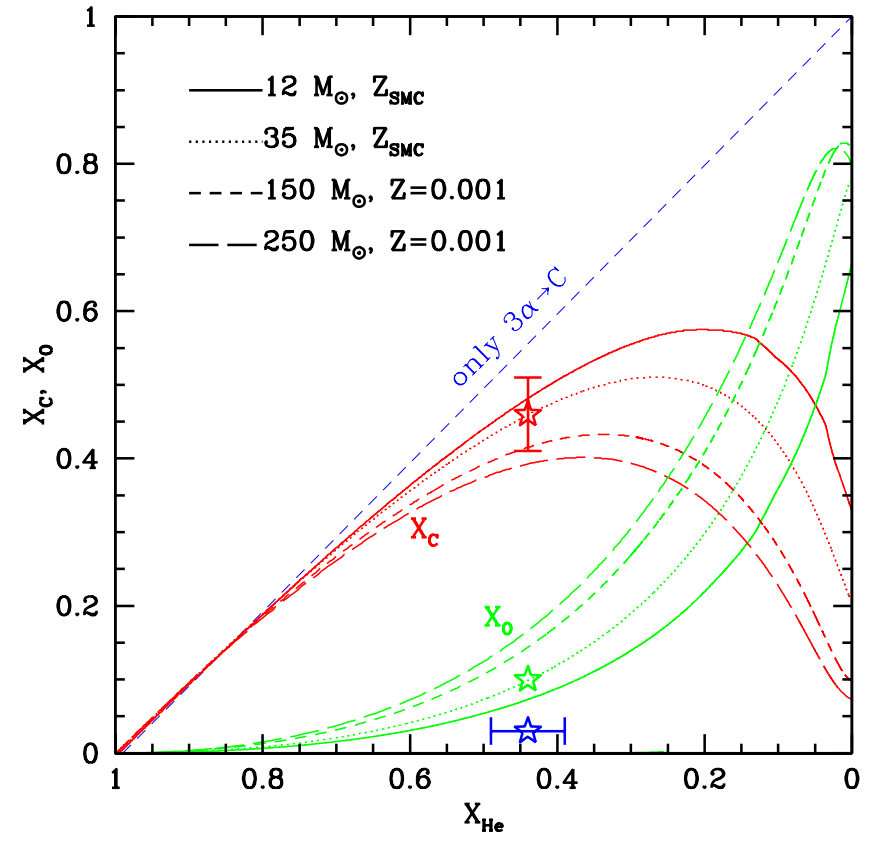

Fig. 11. Comparison of the helium (blue), carbon (red) and oxygen (green) mass fractions of DR1 with evolutionary predictions of heliumburning stars of various masses $\left(12 M_{\odot}\right.$ and $35 M_{\odot}$ from Brott et al. 2011, $150 M_{\odot}$ and $250 M_{\odot}$ from Langer et al. 2007). The uncertainty of the oxygen abundance is comparable to the size of the symbol and therefore not indicated.

DR1 must indeed be well advanced in its core-helium burning stage. Assuming a current mass of $20 M_{\odot}$, the radiative envelope is expected to be $\approx 4.7 M_{\odot}$ (Langer 1989). Based on the mass-loss rate we found for DR1, the envelope is lost at a timescale of at least $\approx 8-9 \times 10^{4}$ years (for an unclumped wind, i.e. $f_{\mathrm{c}}=1$ ). For a helium-burning timescale of $4.3 \times 10^{5}$ year (Langer 1989), we find that DR1 is currently at least three-quarters into core-helium burning. Adopting a probable post-core helium burning lifetime of $10^{4}$ year (Langer 1989), this also suggests that the volume filling factor cannot be much lower than $f_{\mathrm{c}} \approx 0.2$, as otherwise the star should have already exploded. The temperature of DR1 is consistent with the temperature predicted for core-helium burning stars, while it is too low to correspond to post-core helium burning (Langer et al. 1988).

Turning the argument around, the mass-loss rate has obviously been large enough to uncover the material which was inside the convective core in the middle of core-helium burning, i.e. before core-helium burning finished. Adopting the heliumburning timescale given above, this implies that $\dot{M}>4.7 M_{\odot} / \frac{1}{2} \times$ $4.3 \times 10^{5} \mathrm{yr} \approx 2.2 \times 10^{-5} M_{\odot} \mathrm{yr}^{-1}$.

\subsection{Mass-loss rate}

Figure 12 shows a comparison of the mass-loss rate and luminosity of DR1 to the observed values for WC and WO stars in the Galaxy (Sander et al. 2012) and the LMC (Crowther et al. 2000, 2002). The plot clearly shows that the mass-loss rate of WC stars depends on stellar luminosity and the metallicity of the environment. This is in line with model predictions by Vink \& de Koter (2005) and Gräfener \& Hamann (2008), who find that the metallicity dependence of WR mass loss is mainly controlled by the iron abundance. 


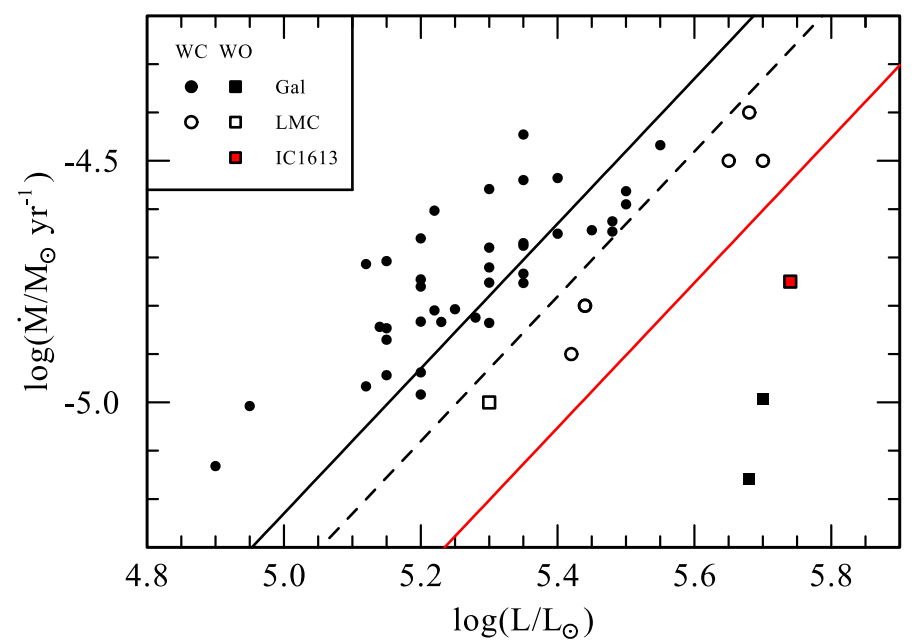

Fig. 12. Comparison of the mass-loss rates and luminosity of WC and WO stars in different metallicity environments to the mass-loss relation WR1 of Yoon \& Langer (2005). The solid black line represents the relation for $Z=Z_{\odot}$, the dashed black line $Z=1 / 2 Z_{\odot}$, and the solid red line $Z=1 / 7 Z_{\odot}$. The solid symbols represent the results from Sander et al. (2012), the open symbols represent the results from Crowther et al. $(2000,2002)$, and the red square indicates the location of DR1.

DR1 fits in this picture, as its mass-loss rate is well below the values found for WC stars of similar luminosity in the LMC. The two Galactic WO stars studied by Sander et al. (2012) have surprisingly low mass-loss rates. This could be caused by the modeling of the $\mathrm{O}_{\mathrm{VI}} \lambda \lambda 3811-34$ line, as this requires a low mass-loss rate to prevent the emission from being re-absorbed in the stellar wind. The mass-loss rate of the LMC WO star analyzed by Crowther et al. (2000), who do not fit the O vi $\lambda \lambda 3811-$ 34 emission, is consistent with the metallicity trend.

Alternatively, the low mass-loss rates of the Galactic WO stars may be an indication that these stars do not follow a well-defined dependence on $L$ and $Z$. One of the reasons for this could be their high temperature. Temperature effects for WR stars are predicted by Gräfener \& Hamann (2008), albeit in a different temperature regime. As pointed out by Gräfener et al. (2012), there may exist a dichotomy between the winds of early WR subtypes which are likely driven by the hot iron opacity peak at $150 \mathrm{kK}$, and later subtypes which may have radially inflated envelopes and much cooler winds (cf. also Nugis \& Lamers 2002; Lamers \& Nugis 2002). The existence of such inflated envelopes has been questioned by Petrovic et al. (2006).

Figure 12 also shows scaled-down fits to empirical mass-loss rates by Hamann et al. (1995), as provided by Yoon \& Langer (2005, their relation WR1). The scaling reduces the empirical rates by a factor of six to correct for the effect of clumping. The mass-loss rate is assumed to scale proportional to $Z^{0.5}$. This prescription reproduces the observed luminosity and metallicity dependence of WR mass loss reasonably well, save for the two Galactic WO stars.

\section{4. lonizing flux and nebular properties}

The observed nebular properties indicate that the nebula surrounding DR 1 is one of the most extreme He II emitting regions known. Nevertheless, the properties can be well modeled with DR1 as the dominating ionizing source. Our best stellar model has an ionizing flux ratio of $\log \left(Q_{2} / Q_{0}\right)=-1.5$. This is much lower than the values ranging from -0.3 to -0.9 found in hot WC models (Smith et al. 2002). These models predict that nebular He II emission is likely not observable for these values of $Q_{0}$ and $Q_{2}$, based on a predicted line strength of He II $\lambda 4686 / \mathrm{H} \beta=$ $2.14 q_{2} / q_{0}$ (Schaerer \& Vacca 1998). Even though our value of $\log \left(Q_{2} / Q_{0}\right)$ is much lower (a line ratio of $\mathrm{He}$ II $\lambda 4686 / \mathrm{H} \beta=0.07$ is predicted by Schaerer \& Vacca 1998), we can reproduce the observed He II emission by adopting a non-homogeneous density profile. The He II emission is also stronger than predicted because the electron temperature of DR1's surrounding nebula is much higher than used in the predictions $\left(T_{\mathrm{e}}=17.5 \mathrm{kK}\right.$ versus $10 \mathrm{kK})$.

\subsection{Final fate}

The eventual fate of DR1 is mostly determined by its mass prior to supernova explosion. Stars with a final mass $>10 M_{\odot}$ are likely to form black holes (e.g., Georgy et al. 2009), producing a faint supernova or no supernova at all. If rapidly rotating, however, it is possible that such massive stars produce a bright type $\mathrm{Ib} / \mathrm{c}$ supernova, possibly with an associated gamma-ray burst (e.g., Woosley \& Bloom 2006). This scenario is more likely at low metallicities, as the mass-loss rates are lower during the various stages of evolution, reducing the angular momentum loss. The massive core can therefore more easily retain the angular momentum needed to power the explosion.

Sander et al. (2012) suggest that the two Galactic WO stars in their analysis have high rotational velocities $\left(\approx 1000 \mathrm{~km} \mathrm{~s}^{-1}\right)$ based on the shape of the lines. If this is indeed the case, these stars would be potential progenitors of GRBs. Even though the line shapes of DR1 can be well fitted by a non-rotational model, we cannot exclude that the star is fairly rapidly rotating, as convolving with rotational profiles of up to $500 \mathrm{~km} \mathrm{~s}^{-1}\left(\approx 0.25 v_{\text {crit }}\right)$ has a negligible effect on the line shapes. Larger projected rotational velocities broaden the lines too much to fit our data and can thus be excluded.

Despite the efforts of finding the progenitors of type $\mathrm{Ib} / \mathrm{c} \mathrm{SNe}$ in pre-supernova images, none have been identified so far (e.g., Eldridge et al. 2013). If the progenitors are hot WR stars like DR1, they would be very hard to detect, as despite their high bolometric luminosity, their visual brightness is very low due to their very high temperature (Yoon et al. 2012).

Although there is still a considerable amount of helium present in our DR1 model, this does not exclude a final explosion in the form of a type Ic supernova. In single stars the helium mass fraction at the surface can be as large as 50 per cent without helium being detected in the spectrum of the supernova (Dessart et al. 2011). The surface helium mass fraction of DR1 is below that level (44 per cent).

\section{Summary}

In this paper we have presented a quantitative spectroscopic analysis of DR1, one of only two WO stars known at a SMClike metallicity. We have modeled the X-Shooter spectrum using CMFGEN in order to derive the stellar and wind parameters. Our best-fit model reproduces the strength and shape of all the $\mathrm{He}, \mathrm{O}$ and $\mathrm{C}$ lines in the 320-2000 $\mathrm{nm}$ wavelength range covered by our observations, including the $\mathrm{O}$ VI $\lambda 5290$ line and the O vi $\lambda 5290 / O$ v $\lambda 5592$ ratio. However, our model cannot reproduce the strong $\mathrm{O}$ vi $\lambda \lambda 3811-34$, which is the prime observational diagnostic of the WO spectral type, simultaneously with the rest of the DR1 spectrum. 
We discussed the possibility that a significant part of the $\mathrm{O}$ vi $\lambda \lambda 3811-34$ line flux is originating from a mechanism that is not included in the modeling, for instance X-ray emission produced at (or close to) the base of the wind. Compared to early WC stars, the stellar temperature of DR1 is high, but the surface oxygen abundance is not enhanced. This suggests that the presence of the highly ionized oxygen emission is caused by excitation effects due to the high temperature.

DR1 is embedded in the hottest known He II emitting nebula in the Local Group. The properties of the nebula are consistent with DR1 being the central ionizing source. In particular the high electron temperature of the nebula is suggestive of a very hot central source and a low ambient metallicity. The capacity of DR1 to fully ionize helium may also be relevant for our understanding of the epoch of He II reionization, believed to have occured at redshifts $z \approx 4-2.7$ (e.g., Syphers \& Shull 2013). Although usually attributed to quasars, WO stars like DR1 may also have contributed to the He II ionization.

Our best fit model and the derived oxygen and carbon abundances suggest that DR1 should be seen as a hot (i.e. earliertype) WC star, and that the presence of strong $\mathrm{O}$ vi $\lambda \lambda 3811-34$ emission in WO spectra does not necessarily imply a larger oxygen abundance, hence a more advanced evolutionary stage. This statement is of importance when comparing with evolutionary computations: WO as a spectral type - i.e. defined by the presence of strong $\mathrm{O}$ VI $\lambda \lambda 3811-34$ emission - is not equivalent to the definition of WO stars usually adopted from a theoretical point of view. The latter is based on an enhanced oxygen content (surface abundances $(\mathrm{C}+\mathrm{O}) / \mathrm{He}>1$ by number, e.g. Smith \& Maeder 1991).

DR1 is located in the high-temperature and high-luminosity domain of the HRD. Compared to evolutionary tracks, its location is compatible with the late stages of evolution of stars with an initial mass $>60 M_{\odot}$, although this number is subject to various assumptions in the theoretical tracks. The carbon and oxygen abundances and stellar temperature suggest that DR1 is currently well into its core-helium burning stage. Based on the derived mass-loss rate, the clumping of the stellar wind should be moderate $\left(f_{\mathrm{c}} \gtrsim 0.2\right)$, as otherwise the star should have already exploded.

Although we find that the WO phase likely does not represent a stage of enhanced oxygen abundance compared to WC stars, we do not exclude the possibility that WO stars are in a more advanced evolutionary stage than WC-type Wolf-Rayet stars. The higher temperatures of WO's may be the result of progressive stripping of the outer layers through the radiation driven wind, exposing consecutively hotter layers.

Alternatively, WO and WC stars may be the end products of stars from different initial mass ranges, in which case the question of evolutionary connection between WO and WC stars does not apply. Detailed spectroscopic analyses of more WO and early-WC stars are needed to decide whether the properties of DR1, in particular its high temperature and WC-like oxygen abundance, are representative of the WO stars as a class and to investigate further the nature of the WO's and their possible evolutionary connection with WC stars.

Acknowledgements. S.d.M. acknowledges support through a Hubble Fellowship grant HST-HF-51270.01-A awarded by the STScI, operated by AURA, Inc., under contract NAS 5-26555 and a Einstein Fellowship grant PF3-140105 awarded by the Chandra X-ray Center, operated SAO under the contract NAS8-03060.

\section{Appendix A: Model atoms}

Table A.1. Overview of the model atoms used.

\begin{tabular}{|c|c|c|c|}
\hline Species & $N_{\mathrm{i}}$ & $N_{\mathrm{s}}$ & $N_{\mathrm{t}}$ \\
\hline $\mathrm{He}_{\mathrm{I}}$ & 27 & 27 & 27 \\
\hline He II & 13 & 13 & 30 \\
\hline $\mathrm{C}_{\text {II }}$ & 22 & 22 & 22 \\
\hline $\mathrm{C}_{\text {III }}$ & 44 & 100 & 243 \\
\hline Civ & 59 & 59 & 64 \\
\hline $\mathrm{O}_{\text {II }}$ & 3 & 3 & 3 \\
\hline O III & 79 & 79 & 115 \\
\hline O IV & 53 & 53 & 72 \\
\hline $\mathrm{Ov}$ & 75 & 75 & 152 \\
\hline OvI & 25 & 25 & 31 \\
\hline $\mathrm{Ne}_{\text {II }}$ & 0 & 42 & 242 \\
\hline $\mathrm{Ne}$ III & 10 & 40 & 182 \\
\hline $\mathrm{Ne}$ IV & 10 & 45 & 355 \\
\hline $\mathrm{Ne} v$ & 10 & 37 & 166 \\
\hline $\mathrm{Ne}$ vi & 10 & 36 & 202 \\
\hline $\mathrm{Ne}$ VII & 10 & 38 & 182 \\
\hline $\mathrm{Ne}$ VIII & 24 & 24 & 47 \\
\hline Si iv & 10 & 37 & 48 \\
\hline $\mathrm{Siv}$ & 10 & 33 & 71 \\
\hline Si vi & 20 & 42 & 132 \\
\hline PIV & 0 & 36 & 178 \\
\hline $\mathrm{Pv}_{\mathrm{v}}$ & 0 & 16 & 62 \\
\hline$S_{\text {III }}$ & 0 & 13 & 28 \\
\hline $\mathrm{S}_{\text {IV }}$ & 0 & 51 & 142 \\
\hline $\mathrm{S}_{\mathrm{v}}$ & 0 & 31 & 98 \\
\hline S VI & 28 & 28 & 58 \\
\hline $\mathrm{Cl}_{\text {IV }}$ & 0 & 40 & 129 \\
\hline $\mathrm{Clv}$ & 0 & 26 & 80 \\
\hline $\mathrm{ClvI}$ & 0 & 18 & 44 \\
\hline $\mathrm{Cl}_{\text {vII }}$ & 0 & 17 & 28 \\
\hline Ar III & 0 & 32 & 346 \\
\hline Ar IV & 0 & 50 & 382 \\
\hline $\operatorname{Arv}$ & 0 & 64 & 376 \\
\hline Ar vI & 0 & 21 & 81 \\
\hline Ar VII & 0 & 30 & 72 \\
\hline Ar VIII & 0 & 28 & 52 \\
\hline $\mathrm{Ca}$ III & 0 & 33 & 110 \\
\hline Ca Iv & 0 & 34 & 193 \\
\hline $\mathrm{Cav}$ & 0 & 45 & 121 \\
\hline Ca vi & 0 & 47 & 108 \\
\hline Ca viI & 0 & 48 & 288 \\
\hline Ca viII & 0 & 45 & 296 \\
\hline Ca IX & 0 & 39 & 162 \\
\hline $\mathrm{Cax}$ & 27 & 27 & 59 \\
\hline Criv & 0 & 29 & 234 \\
\hline $\mathrm{Crv}$ & 0 & 30 & 223 \\
\hline Crvi & 0 & 30 & 215 \\
\hline Mn IV & 0 & 39 & 464 \\
\hline Mn v & 0 & 16 & 80 \\
\hline Mn VI & 0 & 23 & 181 \\
\hline Mn VII & 0 & 20 & 203 \\
\hline
\end{tabular}

Notes. $N_{\mathrm{i}}$ is the number of levels that are treated with an accelerated lambda iteration. $N_{\mathrm{s}}$ is the number of superlevels, each of which may consist of a single level or multiple levels. $N_{\mathrm{t}}$ the total number of atomic levels in the model atom. 
Table A.1. continued.

\begin{tabular}{|c|c|c|c|}
\hline Species & $N_{\mathrm{i}}$ & $N_{\mathrm{s}}$ & $N_{\mathrm{t}}$ \\
\hline $\mathrm{Fe}$ IV & 51 & 51 & 294 \\
\hline $\mathrm{Fe} v$ & 47 & 47 & 191 \\
\hline $\mathrm{Fe}$ VI & 44 & 44 & 433 \\
\hline Fe vII & 41 & 41 & 252 \\
\hline Fe VIII & 53 & 53 & 324 \\
\hline $\mathrm{Fe}_{\text {IX }}$ & 52 & 52 & 490 \\
\hline $\mathrm{Fe} x$ & 43 & 43 & 210 \\
\hline $\mathrm{Ni}$ IV & 0 & 36 & 200 \\
\hline $\mathrm{Niv}$ & 0 & 46 & 183 \\
\hline Ni vi & 0 & 37 & 314 \\
\hline Ni VII & 0 & 37 & 308 \\
\hline Ni VIII & 0 & 34 & 325 \\
\hline Ni IX & 0 & 34 & 363 \\
\hline
\end{tabular}

\section{References}

Armandroff, T. E., \& Massey, P. 1991, AJ, 102, 927

Asplund, M., Grevesse, N., Sauval, A. J., \& Scott, P. 2009, ARA\&A, 47, 481

Baldwin, J. A., Ferland, G. J., Martin, P. G., et al. 1991, ApJ, 374, 580

Barlow, M. J., \& Hummer, D. G. 1982, in Wolf-Rayet Stars: Observations, Physics, Evolution, eds. C. W. H. De Loore, \& A. J. Willis, IAU Symp., 99, 387

Bresolin, F., Urbaneja, M. A., Gieren, W., Pietrzyński, G., \& Kudritzki, R.-P. 2007, ApJ, 671, 2028

Brott, I., Evans, C. J., Hunter, I., et al. 2011, A\&A, 530, A116

Castelli, F., \& Kurucz, R. L. 2004, IAU Symp. 210, poster A20

Crowther, P. A., De Marco, O., \& Barlow, M. J. 1998, MNRAS, 296, 367

Crowther, P. A., Fullerton, A. W., Hillier, D. J., et al. 2000, ApJ, 538, L51

Crowther, P. A., Dessart, L., Hillier, D. J., Abbott, J. B., \& Fullerton, A. W. 2002, A\&A, 392, 653

Crowther, P. A., Morris, P. W., \& Smith, J. D. 2006, ApJ, 636, 1033

Davidson, K., \& Kinman, T. D. 1982, PASP, 94, 634

De Marco, O., Schmutz, W., Crowther, P. A., et al. 2000, A\&A, 358, 187

de Mink, S. E., Langer, N., Izzard, R. G., Sana, H., \& de Koter, A. 2013, ApJ, 764,166

Dessart, L., Crowther, P. A., Hillier, D. J., et al. 2000, MNRAS, 315, 407

Dessart, L., Hillier, D. J., Livne, E., et al. 2011, MNRAS, 414, 2985

D’Odorico, S., \& Rosa, M. 1982, A\&A, 105, 410

Ekström, S., Georgy, C., Eggenberger, P., et al. 2012, A\&A, 537, A146

Eldridge, J. J., Fraser, M., Smartt, S. J., Maund, J. R., \& Crockett, R. M. 2013, MNRAS, 436, 774

Ferland, G. J., Porter, R. L., van Hoof, P. A. M., et al. 2013, Rev. Mex. Astron. Astrofis., 49, 137

Fitzpatrick, E. L. 1999, PASP, 111, 63

Garcia, M., Herrero, A., Vicente, B., et al. 2009, A\&A, 502, 1015

Garnett, D. R., Kennicutt, Jr., R. C., Chu, Y.-H., \& Skillman, E. D. 1991, ApJ, 373,458

Georgy, C., Meynet, G., Walder, R., Folini, D., \& Maeder, A. 2009, A\&A, 502, 611
Gräfener, G., \& Hamann, W.-R. 2008, A\&A, 482, 945

Gräfener, G., Hamann, W.-R., Hillier, D. J., \& Koesterke, L. 1998, A\&A, 329, 190

Gräfener, G., Koesterke, L., \& Hamann, W.-R. 2002, A\&A, 387, 244

Gräfener, G., Vink, J. S., de Koter, A., \& Langer, N. 2011, A\&A, 535, A56

Gräfener, G., Owocki, S. P., \& Vink, J. S. 2012, A\&A, 538, A40

Hamann, W.-R., Koesterke, L., \& Wessolowski, U. 1995, A\&A, 299, 151

Hartoog, O. E., Sana, H., de Koter, A., \& Kaper, L. 2012, MNRAS, 422, 367

Hillier, D. J., \& Miller, D. L. 1998, ApJ, 496, 407

Hillier, D. J., \& Miller, D. L. 1999, ApJ, 519, 354

Ishii, M., Ueno, M., \& Kato, M. 1999, PASJ, 51, 417

Kehrig, C., Oey, M. S., Crowther, P. A., et al. 2011, A\&A, 526, A128

Kingsburgh, R. L., \& Barlow, M. J. 1995, A\&A, 295, 171

Kingsburgh, R. L., Barlow, M. J., \& Storey, P. J. 1995, A\&A, 295, 75

Lamers, H. J. G. L. M., \& Nugis, T. 2002, A\&A, 395, L1

Langer, N. 1989, A\&A, 210, 93

Langer, N., Kiriakidis, M., El Eid, M. F., Fricke, K. J., \& Weiss, A. 1988, A\&A 192, 177

Langer, N., Norman, C. A., de Koter, A., et al. 2007, A\&A, 475, L19

Maeder, A. 1983, A\&A, 120, 113

Meynet, G., \& Maeder, A. 2003, A\&A, 404, 975

Meynet, G., \& Maeder, A. 2005, A\&A, 429, 581

Modigliani, A., Goldoni, P., Royer, F., et al. 2010, in SPIE Conf. Ser., 7737

Neugent, K. F., Massey, P., \& Morrell, N. 2012, AJ, 144, 162

Nomoto, K., Tanaka, M., Tominaga, N., \& Maeda, K. 2010, New Astron. Rev., 54,191

Nugis, T., \& Lamers, H. J. G. L. M. 2002, A\&A, 389, 162

Osterbrock, D. E., \& Ferland, G. J. 2006, Astrophysics of gaseous nebulae and active galactic nuclei (University Science Books)

Osterbrock, D. E., Tran, H. D., \& Veilleux, S. 1992, ApJ, 389, 305

Pagel, B. E. J., Simonson, E. A., Terlevich, R. J., \& Edmunds, M. G. 1992, MNRAS, 255, 325

Petrovic, J., Pols, O., \& Langer, N. 2006, A\&A, 450, 219

Pietrzyński, G., Gieren, W., Soszyński, I., et al. 2006, ApJ, 642, 216

Rubin, R. H., Simpson, J. P., Haas, M. R., \& Erickson, E. F. 1991, ApJ, 374, 564

Rubin, R. H., Dufour, R. J., \& Walter, D. K. 1993, ApJ, 413, 242

Sana, H., de Mink, S. E., de Koter, A., et al. 2012, Science, 337, 444

Sana, H., de Koter, A., de Mink, S. E., et al. 2013, A\&A, 550, A107

Sander, A., Hamann, W.-R., \& Todt, H. 2012, A\&A, 540, A144

Schaerer, D., \& Vacca, W. D. 1998, ApJ, 497, 618

Schlegel, D. J., Finkbeiner, D. P., \& Davis, M. 1998, ApJ, 500, 525

Smartt, S. J., Crowther, P. A., Dufton, P. L., et al. 2001, MNRAS, 325, 257

Schmutz, W., Hamann, W.-R., \& Wessolowski, U. 1989, A\&A, 210, 236

Smith, L. F., \& Maeder, A. 1991, A\&A, 241, 77

Smith, L. J., Norris, R. P. F., \& Crowther, P. A. 2002, MNRAS, 337, 1309

Syphers, D., \& Shull, J. M. 2013, ApJ, 765, 119

Tautvaišienè, G., Geisler, D., Wallerstein, G., et al. 2007, AJ, 134, 2318

Tur, C., Heger, A., \& Austin, S. M. 2007, ApJ, 671, 821

Vernet, J., Dekker, H., D’Odorico, S., et al. 2011, A\&A, 536, A105

Vink, J. S., \& de Koter, A. 2005, A\&A, 442, 587

Vink, J. S., de Koter, A., \& Lamers, H. J. G. L. M. 2001, A\&A, 369, 574

Vink, J. S., Muijres, L. E., Anthonisse, B., et al. 2011, A\&A, 531, A132

Weaver, T. A., \& Woosley, S. E. 1993, Phys. Rep., 227, 65

Woosley, S. E., \& Bloom, J. S. 2006, ARA\&A, 44, 507

Yoon, S.-C., \& Langer, N. 2005, A\&A, 443, 643

Yoon, S.-C., Gräfener, G., Vink, J. S., Kozyreva, A., \& Izzard, R. G. 2012, A\&A, 544, L11

Zaritsky, D., Kennicutt, Jr., R. C., \& Huchra, J. P. 1994, ApJ, 420, 87 